\title{
Evolution Mechanism of Advanced Equipment Manufacturing Innovation Network Structure from the Perspective of Complex System
}

\author{
Jianbo Wang ${ }^{1,2}$ and Xing Cao $\mathbb{i D}^{1,3}$ \\ ${ }^{1}$ Business School, Central South University, Changsha 410083, China \\ ${ }^{2}$ Hunan University of Humanities, Science and Technology, Loudi 417000, China \\ ${ }^{3}$ Hunan First Normal University, Changsha 410205, China \\ Correspondence should be addressed to Xing Cao; caoxingsxy418@csu.edu.cn
}

Received 23 October 2020; Revised 21 November 2020; Accepted 14 December 2020; Published 6 January 2021

Academic Editor: Abd E.I.-Baset Hassanien

Copyright ( 2021 Jianbo Wang and Xing Cao. This is an open access article distributed under the Creative Commons Attribution License, which permits unrestricted use, distribution, and reproduction in any medium, provided the original work is properly cited.

\begin{abstract}
Our country's equipment manufacturing industry ranks among the best in all developing countries, but compared with developed countries, there is still a long way to go. It is not only the backwardness of various technologies, but also the interference of other countries. Although our country's equipment manufacturing industry is not as advanced as the advanced technology of developed countries, we still have to stick to our original aspirations, do not underestimate ourselves, and be good at absorbing and learning from the strengths of others to make up for our own weaknesses. While not working behind closed doors and while absorbing technology from other countries, we can make use of our strengths to make up for our weaknesses and develop our own industrial technology. This paper studies the evolution trend of innovation network structure and at the same time studies the evolution mechanism of advanced equipment manufacturing innovation network structure from the perspective of complex systems. The explained variable in this article is green total factor productivity. The variable adopts the Malmquist-Luenberger global superefficiency index model. There are two main explanatory variables. One is the heterogeneity that affects the efficiency of industrial evolution, including factor heterogeneity, structural heterogeneity, and environmental heterogeneity, and the other is the interaction term of equipment manufacturing specialization agglomeration degree dummy variable multiplied by factor heterogeneity. The regional economic development level is added to the model as a control variable. In the selection of measurement indicators, the per capita GDP is used as the control variable. The experimental results show that each sample is tested in pairs, and the standard error level of the mean is 0.018 , which is less than 0.05 , indicating that the efficiency of the equipment manufacturing industry's economic correlation spatial network has a significant impact on the overall economic development level of the industry. The reduction in spur helps to increase economic output.
\end{abstract}

\section{Introduction}

In recent years, with the increasing development of network technology and the increasing rise of networked organizations, innovation networks formed by the cooperation of different innovation entities have become a new organizational form for enterprise technological innovation activities. The formation of the innovation network is conducive to the accumulation of innovation resources, the improvement of innovation efficiency, and the transformation of scientific and technological achievements. An effective innovation model that can adapt to the current fierce competition only needs to be based on the innovation resources in the park or region, and in terms of time and quantity, it will be far from meeting the innovation and development needs of the cluster system. It is also necessary to adjust the innovation behavior of industrial parks accordingly, that is, from relying on technology learning and knowledge dissemination among local industrial park members, to actively developing external network 
connections to obtain the required external high-level knowledge resource cluster innovation. Initially, static innovation activities that focused only on acquiring one-time knowledge and technical learning became dynamic innovation activities and finally formed continuous innovation through a series of stages and processes.

The world economy has entered a period of relatively low growth. The economies of large developed countries have fallen into recession, and the economic growth of developing countries has slowed. Developed countries such as European countries and the United States have increased their support for the real economy and implemented the "industrialization" strategy in order to seek in-depth adjustments to the economic structure. New changes have taken place in global economic growth. With the growth of market demand for individualization and adaptability, trade protectionism in some developed countries has increased, and some developing countries have also actively participated in the global industrial division of labor due to their advantages in work and raw materials. Besides, other agents donated, accelerated the transfer of industry and capital, and opened up the international market space. Guan gave a model of the evolution of the innovation network structure from a quantitative perspective and pointed out that the structural evolution of the cluster innovation network is mainly reflected in the addition, edge addition, and reconnection behavior of the cluster innovation network [1]. Piccinno et al. used complex network theory to give three simulation models for the evolution of the innovation network structure and calculated the structural characteristics of the innovation network under the three models with the simulation of technology, capital, and market nodes [2]. Li studied the formation and evolution mechanism of cluster innovation networks for specific industrial clusters [3].

China's economic growth has slowed from high-speed growth to medium-high growth, and downward pressure on the national economy has increased. Improving the quality and efficiency of growth has become the main goal of future economic growth. Some contradictions and problems concealed by the period of rapid economic growth are concentrated, forcing enterprises to accelerate their transformation and upgrading. The traditional factor-based and investment-based models no longer meet the requirements of the new economic normal, and the structural reform of the supply side has become a new trend. In the new economic situation, speeding up the transformation of the economic structure and industrial upgrading must have the rise and support of a number of emerging industries characterized by high technology. Hertem et al. conducted an empirical study on the innovation network of the equipment manufacturing industry cluster in Shenyang, but it mainly focused on the node elements and connection mode elements of the equipment manufacturing industry cluster innovation network [4]. Karlik and Platonov analyzed the composition of the equipment manufacturing cluster innovation network from a static perspective and described the innovation network [5]. Zhong summarized and analyzed the structural characteristics of the cluster innovation network, took the typical three types of industrial clusters in the west as an example, and studied the structural characteristics of the cluster innovation network [6].

There is no doubt that China is a large producer and a global factory, and it has always provided a large number of industrial products to the world. However, this situation is in exchange for a large amount of cheap labor in our country. The current growth model has reached its limit, and other ways must be found to rescue the manufacturing industry. The novelty of this article lies in the application of entropy method to the innovation and development evaluation of equipment manufacturing industry. Through the research and analysis of the development status of the equipment manufacturing industry and the factors affecting its innovation and development, an evaluation index system for the innovation development of the equipment manufacturing industry has been established, and an empirical analysis of the innovation development of the equipment manufacturing industry has been carried out.

\section{Evolution Mechanism of Advanced Equipment Manufacturing Innovation Network Structure from the Perspective of Complex System}

2.1. Connotation and Characteristics of Equipment Manufacturing Cluster. The equipment manufacturing cluster is different from the general cluster and has its own uniqueness. However, from the current domestic research, there is currently no unified concept. Different scholars have given their own opinions from different angles [7]. In order to further analyze the composition of the equipment manufacturing cluster and its innovation network, it will be defined from the perspective of dynamic evolution [8].

\subsubsection{Connotation and Characteristics of Equipment} Manufacturing Industry. According to the definition of relevant domestic research institutions and scholars, the equipment manufacturing industry is also called the equipment industry [9]. It is a general term for industries that manufacture various technical equipment to meet the development of various sectors of the national economy and national security needs. According to the classification of the national financial industry, we can divide the equipment into five categories according to the content of knowledge and the technical difficulty of the product, supplemented by the needs of direct control by the country: general equipment, basic equipment, basic hightech equipment, complete equipment, and safety equipment [10]. Compared to the light processing industry, the main features of the equipment manufacturing industry are complex technical composition, a variety of supporting elements, large companies with strong technological capabilities of integration and innovation, and higher capital and labor requirements. With the process of global integration, global cooperation in technology research, development, production, and sales is increasingly strengthened. 
2.1.2. Composition of Equipment Manufacturing Cluster Innovation Network. Cluster innovation is a process in which the enterprises in the cluster continuously acquire knowledge from the formal and informal network of relationships in which they are located, and integrate them [11]. The main bodies of the equipment manufacturing cluster have formed various close relationships that contribute to the improvement of innovation capabilities due to their exchanges and connections with each other, and they have obvious network characteristics [12]. The equipment manufacturing cluster innovation network is as follows: under a specific regional social and cultural background, enterprises and related institutions in the equipment manufacturing cluster establish a long-term and relatively stable form that can promote innovation within the cluster through communication with each other.

\subsubsection{Node Elements of Equipment Manufacturing Cluster} Innovation Network. The node elements of the equipment manufacturing cluster innovation network mainly include enterprises, universities/scientific research institutions, intermediary service organizations, and governments [4]. Different from the general cluster innovation network, the center and main body of the equipment manufacturing cluster innovation network are the leading enterprises in the cluster. A large number of small- and medium-sized supporting enterprises have gathered around these leading enterprises. Due to the high technological content of the equipment manufacturing industry, although some large leading companies have mastered a large number of leading technological advantages through introduction, digestion, and absorption, they must have their own proprietary knowledge to obtain sustained competitive advantages [13]. To this end, some large leading companies have also established long-term cooperative relationships with universities, scientific research institutes, and vocational and technical schools within and outside the cluster to expand technological research and development capabilities and enhance independent innovation capabilities. The equipment manufacturing industry is a highly capital-intensive and policyoriented industry, so the government plays an important role in the cluster innovation network. On the one hand, it can help enterprises in the cluster to win more national, provincial, and ministerial projects; on the other hand, it can provide various policy support for cluster enterprises to improve the level of cooperation and social cooperation between cluster entities in the region. The capital stock ensures the efficient transfer of knowledge and information within the cluster.

\subsubsection{Relational Connection of Equipment Manufacturing} Cluster Innovation Network. Similar to the general cluster innovation network, the relationship connection between the subjects includes both formal cooperation agreements and informal exchanges and communication, and with the number of relationships between the subjects, the connection can be strong or weak [14]. Since the difference between equipment manufacturing clusters and general industrial clusters is mainly reflected in the enterprise nodes, this section focuses on the relationship between enterprises and enterprises. In addition to the relational connection of the industrial chain model, there is also a strong competition-cooperative model relational connection between cluster enterprises. Due to the differences in enterprise scale and capabilities, a group of flying geese is usually formed with large enterprises as the core, medium-sized enterprises as the second echelon, and small enterprises as auxiliary. The competition among cluster enterprises is mainly manifested as the grabbing of market and resources among enterprises at the same level [15]. Generally speaking, the number of leading companies in a cluster is small, and there is no strong competitive relationship between them, which is mainly reflected in the plundering of external markets and resources with other companies of the same type outside the cluster. In order to reduce costs and gain a competitive advantage, they have formed a close supporting cooperative relationship with small- and medium-sized supporting enterprises, namely, cooperative relations.

\subsection{Promotion Mechanism of Technological Progress on In-} dustrial Upgrading. Industrial upgrading is a process of continuous development and change based on technological progress. From the perspective of supply, technological advancement promotes industrial upgrading by creating new industries, providing new products, changing factor supply ratios, promoting industrial integration, and improving the quality of human capital, thereby promoting industrial upgrading and thus continuing to increase productivity [16]. The technological structure upgrade and the industrial structure upgrade show a one-to-one correspondence in a relatively long period of time.

\subsubsection{Technological Progress Promotes Industrial Upgrading} by Creating Emerging Industries and Eliminating Backward Industries. Each product must go through the start-up phase, the growth phase, the maturity phase, and the decline phase and finally be replaced by a newer product. The impact of changes in demand structure on the industrial structure is immediate. Technological progress is an important factor in promoting product upgrades and creating new industries [17]. There are two emerging industries in the field of equipment manufacturing: One is an emerging industry, which refers to an industry that has gradually formed due to technological discovery. In an industry that has never been seen before, major technological advances will stimulate new demand for the formation of a new industry [18]. The second is industrial integration, which refers to the formation of new industries by integrating different subindustries within an industry or between different industries on the basis of continuous technological integration. Emerging industries created through technological integration can usually meet market demand and occupy a larger market share.

\subsubsection{Technological Advancement Promotes Industrial} Upgrading by Providing New Tools, New Processes, and New Methods. The three elements of technology are tools, methods, and processes. Technological progress has 
promoted the emergence of new tools, new methods, and new processes; improved the use efficiency of factor inputs; reduced the consumption of energy and resources in the production process; and thus reduced costs. Due to the emergence of new tools, new methods, and new processes, the traditional equipment manufacturing industry can also undergo technological transformation, and its product quality will be improved accordingly [19]. At the same time, affected by this, upstream and downstream enterprises in the industrial chain will also carry out technological upgrades to adapt to the coordinated development of the industrial chain.

\subsubsection{Technological Progress Promotes Industrial Upgrading} by Changing the Proportion of Factor Input Allocation. The rate input for each subindustry is different from the ratio of the equipment industry. Because each industry has different technical levels and technical capabilities, and the speed and effect of technological progress are also different, the elasticity of demand in each industry will change, leading to demand. The rate input for each subindustry is different from that of the equipment industry. Because each industry has different technical levels and technical capabilities, and the speed and effect of technological progress are also different, the demand elasticity of each industry will change, leading to changes in the demand structure [20]. When technological progress and other reasons increase the market demand for new products, the profitability of capital will push a large number of producers into the new product industry, thereby promoting the internal upgrading of the industry.

2.3. Model Description. In the classic BA model, the premise of its assumption is that there are isolated points in the cluster innovation network. However, from the actual network connection, in addition to formal relationship links, there are also informal exchanges and communication, and there are many types of nodes in the network, so the probability of outliers in the network is very small [21].

On the premise of satisfying complementary resources, any individual first selects the "local world" of the node newly added to the network when contacting other individuals in the network and then contacts other members of the "local world" network. This is the so-called "cluster network", and the probability of preferential connection is

$$
p\left(k_{i}\right)=\frac{k_{i}}{\sum_{j \in N} k_{j}} .
$$

$N$ represents the node set formed by all nodes, $k_{i}$ represents the node degree of node $i$, and $k_{j}$ represents the node degree of node $j$.

Add $m$ new edges to the network with probability $p(0 \leq p<1)$. In the initial network, a node is randomly selected as the starting point, and the other end is selected according to (1). Repeat the process $m\left(0 \leq m<m_{0}\right)$ times. At this time, the degree value of node $i$ is

$$
\left(\frac{\partial k_{i}}{\partial t}\right)=p m \frac{1}{N}+p m \frac{k_{i}}{\sum_{j \in N} k_{j}} .
$$

Add $m$ edges to the network with probability $q(0 \leq p<1)$. First, randomly select an edge of a node. After disconnecting this edge, connect to node $j$, and follow the probability of (1) when selecting $j$. Repeat this process $m$ times. At this time, the degree value of the node $i$ is

$$
\left(\frac{\partial k_{i}}{\partial t}\right)=-q m \frac{1}{N}+q m \frac{k_{i}}{\sum_{j \in N} k_{j}} .
$$

Add a new node with probability $1-p-q(0 \leq p+q<$ $1)$. The new node is connected with the existing $m$ nodes in the graph according to the probability of (1). At this time, the degree value of node $i$ is

$$
\left(\frac{\partial k_{i}}{\partial t}\right)=(1-p-q) m \frac{k_{i}}{\sum_{j \in N} k_{j}} .
$$

From comprehensive formula (2)-(4), we have

$$
\frac{\partial k_{i}}{\partial t}=(p-q) m \frac{1}{N}+m \frac{k_{i}}{\sum_{j \in N} k_{j}},
$$

where $p$ represents the probability of adding edges, $q$ represents the probability of reconnection, and $m$ represents the number of nodes or edges added to the center of the network. $N$ represents the number of nodes in the entire network. As time changes, it will gradually meet the following conditions:

$$
\begin{aligned}
N_{1} & =m_{0}+(1-p-q) t, \\
\sum_{j} k_{j} & =m t(1-p-q)+\sum_{j} k_{j}(0) .
\end{aligned}
$$

When $t$ is large enough, the influence of $m_{0}$ and $\sum_{j} k_{j}(0)$ on $N_{t}$ and $\sum_{j} k_{j}$ is small and can be ignored:

$$
\begin{aligned}
N_{t} & =(1-p-q) t, \\
\sum_{j} k_{j} & =m t(1-p-q) .
\end{aligned}
$$

From the combination of (4) and (8), we can get

$$
\frac{\partial k_{i}}{\partial t}=\frac{1}{t}\left(b+\frac{k_{i}}{1+p-q}\right) .
$$

And because of $\left(\mathrm{d} k_{i} /\left(b+\left(k_{i} / 1+p-q\right)\right)\right)=(\mathrm{d} t / t)$, we can get

$$
k_{i}\left(t_{i}\right)=C(1+p-q) t^{(1 /(1+p-q))}-b(1+p-q) .
$$

Let $k_{i}\left(t_{i}\right)=m$; then,

$$
\begin{aligned}
C & =\frac{m+b(1+p-q)}{(1+p-q) b t_{i}^{(1 /(1+p-q))}} \\
k_{i}(t) & =(m+b(1+p-q))\left(\frac{t}{t_{i}}\right)^{(1 /(1+p-q))}-(1+p-q) b .
\end{aligned}
$$


Among them,

$$
b=\frac{p-q}{1-p-q} m .
$$

In order to concretely describe the evolution of the equipment manufacturing industry cluster innovation network structure, below we will combine the phase characteristics of this evolution.

\subsection{Characteristics of Technological Innovation Network of High-Tech Enterprises}

2.4.1. Highly Complementary Knowledge Resources among Innovative Subjects. The problem of the optimal use of distributed knowledge is certainly not a new problem, it is only caused by the arrival of the knowledge economy, and any complex social system will face it. With the advent of the knowledge economy, the intensity of product knowledge has increased, and there is an increasing need to combine different distributed knowledge from different sources. In the high-tech industry, product knowledge is distributed in the hands of different production companies in the industry, and everyone continues to deepen their knowledge in a specific field. However, when the whole product is produced, it can be integrated with each other. Therefore, the high complementarity of innovation resources is very obvious in the high-tech industry [22]. From another perspective, when high-tech companies choose cooperative innovation partners, they generally examine the two important factors influencing cooperation complementarity and technological leadership. The complementarity of cooperation includes two aspects: one is the complementarity of technical resources, and the other is the complementarity of organization and management. Generally speaking, the more complementary the technical resources of the parties involved in the technological innovation network, the greater the performance produced by the technological innovation network.

2.4.2. Uncertainty in the Development of Technological Innovation Networks. High technology has the characteristics of highly intensive knowledge, so high technology has strong crossover and integration. In the development process, with the help of the original accumulation and innovation of technology, further development and innovation will form a qualitative leap in science and technology under new historical conditions. It is precisely because high technology is at the forefront of science and technology, so any pioneering ideas, design, and implementation methods are uncertain, and many research results are difficult to predict. If the gradual model represents the evolution of quantitative changes, then the transition model means the evolution of qualitative changes. Although the form of the product is inextricably linked with the past, the basic technology is far away [23]. Technological changes are usually caused by two approaches to solve real-world problems or capture new technology and market opportunities. Technological changes usually lead to transitions in technological orbits, and changes in technological orbits usually require new core capabilities. This shows from another perspective that this change in technology will increase the complementary resources in the network, which will lead to the expansion of the technological innovation network to obtain these complementary resources. Corresponding to these processes, the learning of technological innovation network will change, from the previously narrow knowledge field to a broader knowledge and technology field.

2.4.3. Overall Importance of Social Capital. In the embedded network relationship, companies with a large amount of social capital have many information channels, which can attract favorable partners and alliances and negotiate favorable conditions for themselves. The integrated relationship increases the knowledge exchange between companies, which is the basis for maintaining the partnership. Interinstitutional trust is a prerequisite and an important stimulus for knowledge exchange and innovation. This process requires the support of social capital from high-tech companies. Collective learning transports knowledge to space, which is a dynamic process of free knowledge transfer between entities [24]. Collective learning is a process of knowledge flow caused by the interaction between organizations in a network of technological innovation and a social process of knowledge accumulation. This process is based on the rules and procedures followed by all members of the network, which can go beyond individual rationality and draw on collective logic. The main body of network innovation responds to the uncertainties faced by the technological innovation network through collective observation, evaluation, and coherent measures (Figure 1).

\section{Evolution Mechanism of Advanced Equipment Manufacturing Innovation Network Structure from the Perspective of Complex Systems}

3.1. Demand Analysis. The "independent" upgrade path of system engineering of the model aims not only to increase investment in independent innovation, but more importantly to accurately answer the specific methods of independent innovation to promote industrial upgrading. With the advancement of technology, the enabling role of information technology has been further demonstrated, and it is transforming from information technology supporting business development to information technology leading innovation-driven and transformational development. The theory of technological innovation and industrial upgrading takes the technological accumulation formed by the continuous development of the enterprise as the main driving force and is based on the realization of regional expansion. Due to the improvement of the level of technological development, overseas investment models have gradually shifted from resource acquisition to technology acquisition. In addition, related industries for overseas investment are gradually upgrading, and their composition is related to the 


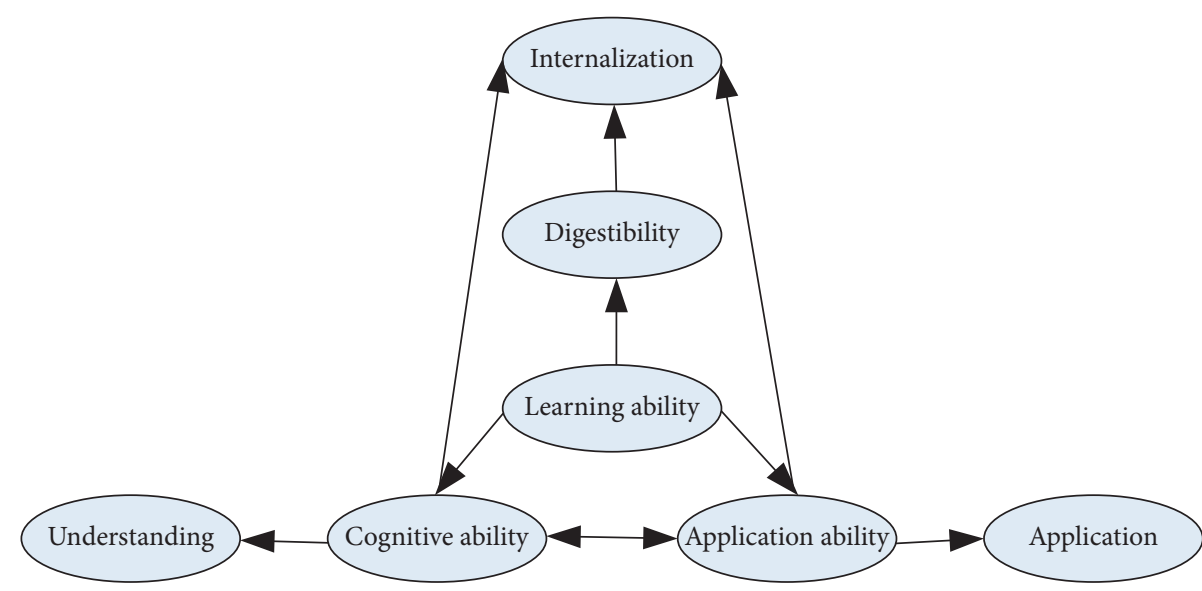

FIgURE 1: Relation diagram of equipment manufacturing cluster innovation network.

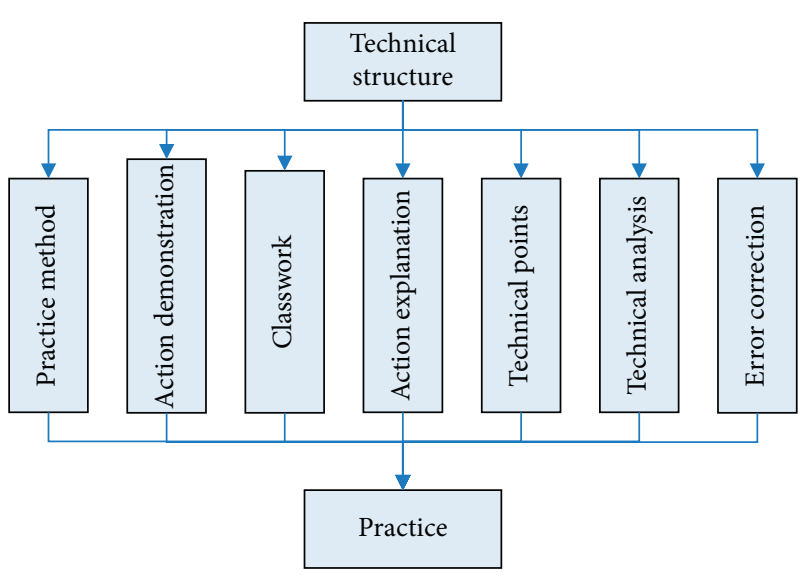

Figure 2: The structural mode of the technical content of gymnastics courseware.

adjustment of regional location distribution. It is of great significance for emerging countries to use overseas investment to achieve technological progress and upgrades, thereby further optimizing the industrial structure and increasing the level of international competition. The overall framework of system requirements is shown in Figure 2.

3.2. Test Subject. The variables studied in this experiment mainly include three categories: explained variables, main explanatory variables, and control variables. The explained variable in this paper is green total factor productivity, and the variable uses the Malmquist-Luenberger global superefficiency index model. There are two main explanatory variables. One is the heterogeneity that affects the efficiency of industrial evolution, including factor heterogeneity, structural heterogeneity, and environmental heterogeneity, and the other is the virtual variable and factor of the equipment manufacturing industry's specialization concentration. The interaction terms of heterogeneity multiply, the level of regional economic development is added to the model as the control variable, and the per capita GDP is used as the control variable in the selection of measurement indicators. The improvement of green total factor productivity is closely related to the level of regional economic development. Generally speaking, a high level of regional economic development will bring economic support to the green development of the equipment manufacturing industry, such as sound infrastructure and a large amount of consumption, demand, etc. In addition, the ability to introduce or develop advanced eco-environmental protection technologies and carry out larger-scale product and service innovations is conducive to promoting the rapid growth of green total factor productivity.

3.3. Model Building. The integration of vehicle control skills has two situations, promoting effect and hindering effect on the total factor productivity of China's equipment manufacturing industry. Considering the nonlinear relationship between vehicle control skill integration index and total factor productivity, this paper introduces the square term of integration index to analyze the nonlinear curve relationship under the influence of the two effects. This paper adds industry variables such as $R \& D$ investment intensity, property rights characteristic factors, export dependence, condition construction intensity, number of highquality scientific and technological personnel, and time variables as control variables to control the influence of industry characteristics and time trends. In order to enable one-to-one correspondence of data, this paper merges the general equipment manufacturing industry and special equipment manufacturing industry into general and special equipment manufacturing industries. Therefore, all control variables are merged into 6 industries. The classification standards are the same.

3.4. Data Processing. The index selected in this article is the annual statistical data of books, which is the index data of practical value. Therefore, the trapezoidal fuzzy table distribution function is usually used to calculate the participation of each indicator. The specific formula is as follows:

Large trapezoidal distribution: 


$$
r(x)=\frac{x-c}{d-c}, \quad c<x<d .
$$

Small trapezoidal distribution:

$$
r(x)=\frac{b-x}{x-a}, \quad a<x<b .
$$

For the ideal score, it can usually be set to the middle value of each interval. This paper divides the evaluation grades into five grades, and the index data after normalization processing all fall into the [0-1] interval.

There are many data standard processing methods, but different data standardization methods will have a certain impact on the evaluation results of the system. For the positive indicator standardization method,

$$
y_{i j}=\frac{x_{i j}-\min \left\{x_{i j}\right\}}{\max \left\{x_{i j}\right\}-\min \left\{x_{i j}\right\}} .
$$

For the negative index standardization method,

$$
y_{i j}=\frac{\max \left\{x_{i j}\right\}-x_{i j}}{\max \left\{x_{i j}\right\}-\min \left\{x_{i j}\right\}} .
$$

After standardizing the data, using the principal component analysis of nonlinear logarithmic centering, the processing steps of logarithmic transformation and row vector centering are

$$
z_{i j}=\ln y_{i j}-\sum_{i=1}^{m} \frac{\ln y_{i j}}{m} .
$$

3.5. Statistical Methods. SPSS 23.0 software was used for data processing, and the count data was expressed in percentage (\%); $k$ is the amount of data in this experiment, $\sigma^{2}$ is the variance of all survey results, and $P<0.05$ indicates that the difference is statistically significant. The formula for calculating reliability is shown in

$$
a=\frac{k}{k-1}\left(1-\frac{\sum \sigma_{i}^{2}}{\sigma^{2}}\right) \text {. }
$$

\section{Evolution Mechanism of Advanced Equipment Manufacturing Innovation Network Structure from the Perspective of Complex System}

\subsection{Evaluation Index System Based on Index Reliability} Testing. Reliability refers to the stability and consistency of the questionnaire. This article adopts the $\alpha$ coefficient method created by Cronbach. The $\alpha$ coefficient can be obtained by Reliability Analysis in SPSS software. It is generally believed that the $\alpha$ coefficient above 0.8 indicates that the effect of the index setting is very good, and above 0.7 is also acceptable. The results are shown in Table 1.
It can be seen from Table 1 that the cluster analysis of the four types of data of completed value added, sales revenue, profits and taxes, and profits in this experiment is acceptable $(\alpha>0.7)$, and there is no absolute difference between the various development stages. The pros and cons are not that the more complex the model, the better the prediction performance. Within the acceptable range, the preconditions for the experiment are met, which provides a basis for subsequent experimental analysis.

4.2. Evolution Trend of Innovation Network Structure. The visualization diagram and small-world measurement of the complex network simulation of the innovation network structure can only have a preliminary grasp and understanding of the network structure form as a whole. In order to fundamentally recognize and understand the evolution trend of the innovation network structure, this article investigates the network evolution trend from two specific indicators of network density and network relevance.

4.2.1. Evolution Trend of Innovation Network Density. The economic system and the mode of economic growth have established fundamental changes of great significance: one is the shift from a planned economy to a market economy, and the other is the shift from an extensive economy to an intensive economy. The two fundamental changes have greatly promoted the efficiency of the allocation of economic factors among regions and strengthened the links between economic entities. The evolution trend is shown in Figure 3.

It can be seen from Figure 3 that although a higher network density is conducive to strengthening the connection between innovative network structures, a higher density also means more connections, which also causes "redundant lines" in the network. If there are too many redundant connections, this will extend the connection path, increase the transaction cost, reduce the efficiency of resource and element allocation, and inhibit the speed of economic development. Therefore, it is necessary to consider maintaining a suitable network density to ensure the flow speed and configuration efficiency of the elements as much as possible.

4.2.2. Evolution Trend of Innovation Network Relevance. The network correlation characteristics of the comprehensive economic space of the urban agglomeration in the Yangtze River Delta are measured by using the network correlation degree, rank degree, and efficiency in the complex network analysis method. The result is shown in Figure 4.

It can be seen from Figure 4 that the continuous improvement of the market-oriented system and the continuous improvement of the supply and demand system and communication interaction of the factor market have promoted the market's fundamental role in resource allocation and have reduced the transaction costs of the flow and linkage of factors between regions. As a 
TABLE 1: Summary table of reliability test results.

\begin{tabular}{|c|c|c|}
\hline Intelligent analysis & Type of data & Alpha coefficient $(\alpha)$ \\
\hline The embryonic stage of innovation network & $\begin{array}{c}\text { Complete added value } \\
\text { Sales revenue } \\
\text { Profit and tax } \\
\text { Profit }\end{array}$ & 0.8636 \\
\hline The growth period of innovation network & $\begin{array}{l}\text { Complete added value } \\
\text { Sales revenue } \\
\text { Profit and tax } \\
\text { Profit }\end{array}$ & 0.7742 \\
\hline The mature period of innovation network & $\begin{array}{c}\text { Complete added value } \\
\text { Sales revenue } \\
\text { Profit and tax } \\
\text { Profit } \\
\end{array}$ & 0.7384 \\
\hline Innovation network decline or change period & $\begin{array}{c}\text { Complete added value } \\
\text { Sales revenue } \\
\text { Profit and tax } \\
\text { Profit }\end{array}$ & 0.7429 \\
\hline
\end{tabular}

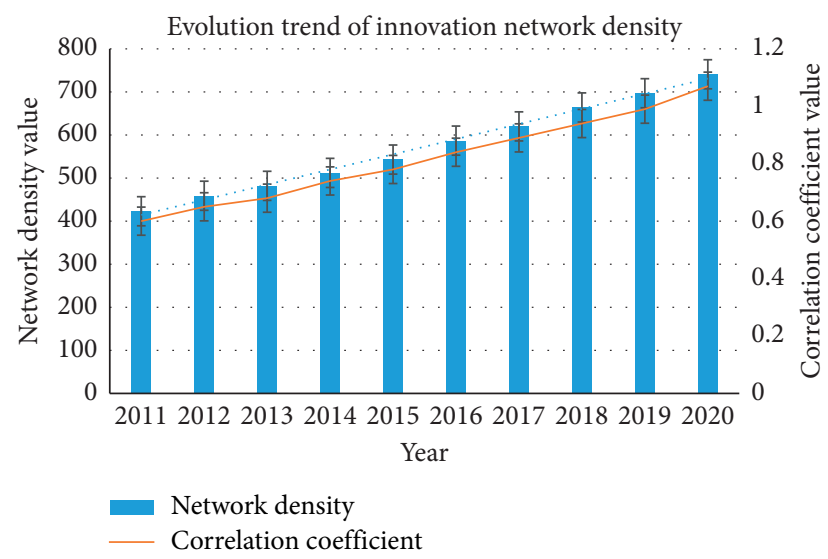

FIgURe 3: Analysis of the evolution trend of innovation network density.

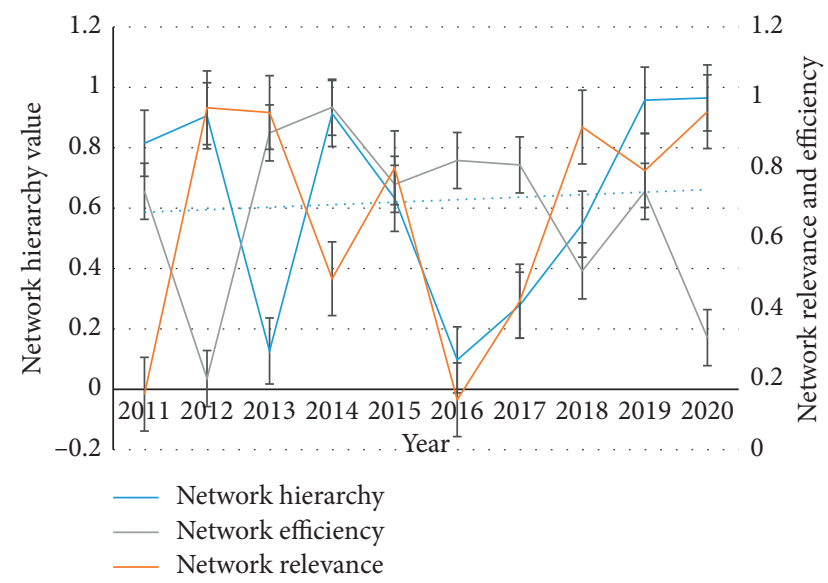

Figure 4: Analysis of the evolution trend of innovation network relevance.

result, the relationship between the economic space of the innovation network structure is increasing, and the stability of the innovation network structure is strengthened.

\subsection{Experimental Evolution of Equipment Manufacturing Cluster Innovation Network Structure}

4.3.1. Development of Equipment Manufacturing Industry Cluster. Through the development in recent years, the scale of small- and medium-sized supporting enterprises in the equipment manufacturing industry in the city has gradually expanded, the industrial concentration and specialization level have been greatly improved, and the scale benefits of industrial clusters have played a role. With the support of all walks of life, the equipment manufacturing industry strives to adjust the industrial structure and improve the quality of operation. The results are shown in Table 2. At the same time, the mean, standard deviation, and standard error of the mean are calculated, as shown in Figure 5.

It can be seen from Figure 5 that at the same time each sample is tested in pairs, and the standard error level of the mean is 0.14 , which is greater than 0.05 , indicating that the network density of the comprehensive economic correlation spatial network of the equipment manufacturing industry has a significant impact on the overall economic development level of the industry. The increase in network density can significantly increase economic output. It can be seen from Table 2 that, from the perspective of increasing the density of the network, in order to achieve an increase in economic output and rapid economic development, we should continue to attach importance and give full play to the fundamental role of the market in the allocation of resources and factors, and promote the spatial flow of factors.

4.3.2. Evolution of Equipment Manufacturing Innovation Network Structure Based on Logistic Algorithm. By adopting single-factor and multifactor control variable method, the equipment manufacturing innovation network structure model based on binomial logistic regression algorithm is established. In the process of parameter tuning, it mainly adjusts the maximum number of iterations of the model, the learning rate, and the maximum depth of the tree. 
TABLE 2: Equipment manufacturing industry cluster development.

\begin{tabular}{lcccc}
\hline Year & Complete added value & Sales revenue & Profit and tax & Profit \\
\hline 2015 & 400.6 & 249.81 & 29.02 & 40.42 \\
2016 & 460.3 & 324.78 & 21.94 & 60.1 \\
2017 & - & 390.78 & 33.01 & 80.34 \\
2018 & 570.9 & - & 121.2 & - \\
2019 & 630.8 & - & 93.4 & - \\
2020 & 700.5 & - & - \\
\hline
\end{tabular}

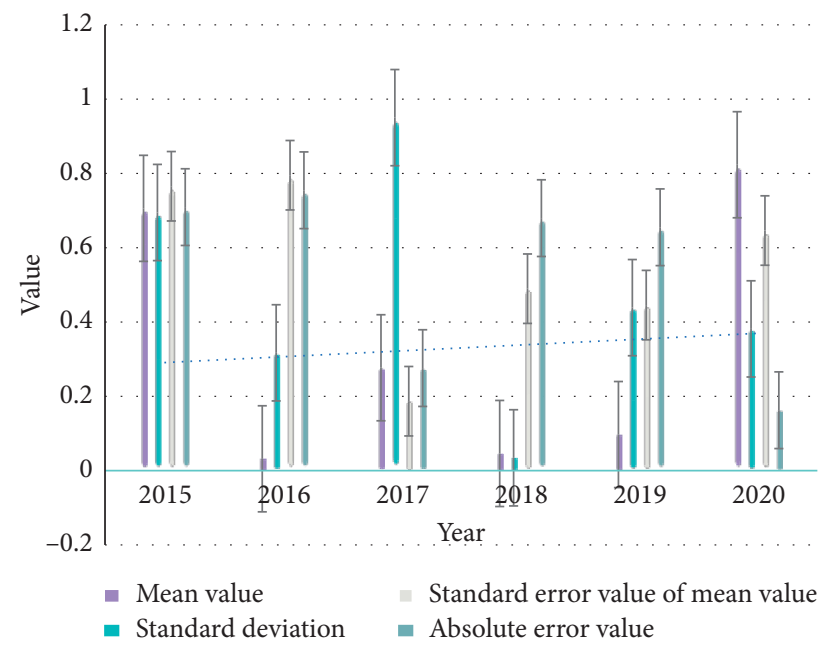

FIgURE 5: Analysis chart of equipment manufacturing cluster development.

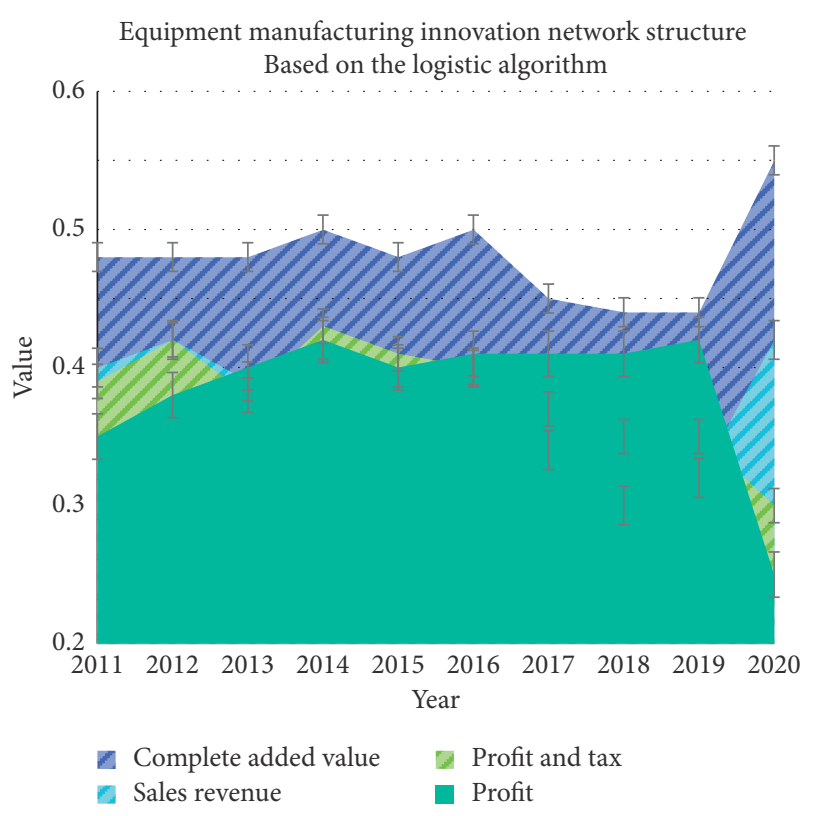

Figure 6: Analysis diagram of the evolution of the equipment manufacturing innovation network structure based on the logistic algorithm.

Each iteration will produce a weak learner. If the number of weak learners is too small, it is easy to underfit, and if there are too many, it is easy to overfit. The result is shown in Figure 6. At the same time, each sample is tested on the selected samples, and data such as the mean, standard deviation, and standard error of the mean are obtained, as shown in Table 3.

It can be seen from Table 3 that at the same time each sample is tested in pairs, and the standard error value of the mean is 0.004 , which is less than 0.05 . The network level of the economic correlation spatial network of the equipment manufacturing industry has a significant impact on the overall economic development level of the industry. The reduction of network level will help increase the economic output of the equipment manufacturing industry. It can be seen from Figure 6 that the decline in the level of the network means that the level of the network's hierarchical structure becomes smaller; that is, the subordinate status of the equipment manufacturing industry's economy has changed from the subordinate status of the one-way connection in the past to the equal status of the two-way connection.

\subsubsection{Evolution of Equipment Manufacturing Innovation} Network Structure Based on MMD Algorithm. By adopting single-factor and multifactor control variable methods, the equipment manufacturing innovation network structure model based on MMD algorithm is established. In the process of parameter tuning, it mainly adjusts the maximum number of iterations of the model, the learning rate, and the maximum depth of the tree. Each iteration will produce a weak learner. If the number of weak learners is too small, it is easy to underfit, and if there are too many, it is easy to overfit. The result is shown in Figure 7. At the same time, each 
TABLE 3: Analysis table of the results of the risk assessment system of Yebes algorithm.

\begin{tabular}{lccc}
\hline & Mean & Standard deviation & Standard error of the mean \\
\hline Complete added value & 2.32 & 9.462 & 1.526 \\
Sales revenue & 1.94 & 8.347 & 1.524 \\
Profit and tax & -1.46 & 9.432 & 1.529 \\
Profit & -1.21 & 8.462 & 1.527 \\
\hline
\end{tabular}

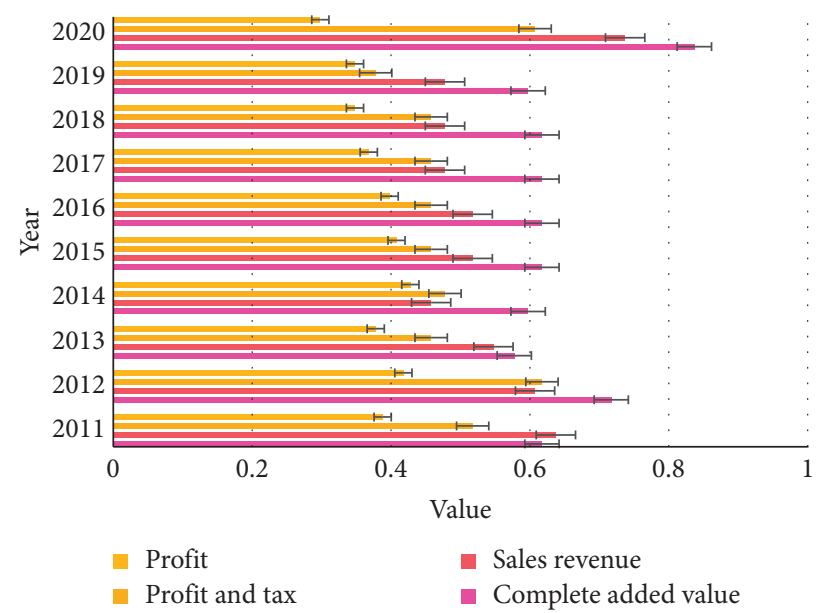

FIGURE 7: Research diagram of the evolution of the equipment manufacturing innovation network structure based on the MMD algorithm.

TABLE 4: Analysis table of the results of MMD algorithm risk assessment system.

\begin{tabular}{lccc}
\hline & Mean & Standard deviation & Standard error of the mean \\
\hline Complete added value & 1.49 & 11.624 & 1.824 \\
Sales revenue & 1.42 & 11.523 & 1829 \\
Profit and tax & -1.33 & 9.643 & 1.276 \\
Profit & -1.01 & 9.427 & 1.271 \\
\hline
\end{tabular}

sample is tested on the selected samples, and data such as the mean, standard deviation, and standard error of the mean are obtained, as shown in Table 4.

It can be seen from Table 4 that at the same time each sample is tested in pairs, and the standard error level of the mean is 0.018 , which is less than 0.05 , indicating that the network efficiency of the equipment manufacturing industry's economic correlation spatial network has a significant impact on the overall economic development level of the industry. The reduction of network efficiency helps to promote the increase of economic output. It can be seen from Figure 7 that we will continue to accelerate the integrated construction of the equipment manufacturing industry, further give full play to the decisive role of the market in resource allocation, strengthen the supply and demand links between parts, reduce the cost of economic space interaction, and gradually improve the stability of the spatial connection network.

\section{Conclusions}

From the perspective of network structure, China's equipment industry innovation network presents an example of a "core-periphery" structure. There are mainly scattered parallel structures, star-shaped dominant structures, and cooperative composite structures. Most of them do not have a coordinated complex structure. This shows that the current innovation network structure of China's equipment industry is unified and lacks stability. Establishing a multilevel collaborative innovation system and strengthening the innovation network of large companies have become necessary means to promote the development of the equipment industry. From the perspective of network characteristics, the current innovation network of China's equipment industry has low density, poor coherence, lack of central organization, insufficient overall network control, and low knowledge transfer efficiency. State-owned enterprises are the core of the network and continue to be the most important part of the equipment industry. The deficiencies of state-owned enterprises have also led to lagging innovation; private companies and consortia have limited flexibility and innovation capabilities, while universities and research institutions are at the edge of the network, and their contribution to innovation is low. Optimizing the industrial structure and exerting the vitality of different components constitute the way to future development.

Large infrastructure equipment companies must act as technology portals and importers of industrial complex 
knowledge; establish external network relationships through active strategic activities, thereby introducing advanced external knowledge and technology into the cluster system; group through the formation of crossregional and cross-border alliance organizations; carry out cross-border regional exchanges and cooperation; organize and coordinate external network contacts; and promote the interaction and exchange of innovative resources. On the one hand, we need to guide large infrastructure equipment companies to promote exchanges and cooperation through direct investment; policy support, and innovation platforms; stimulate innovative production; and narrow the knowledge gap with the outside to improve technological capabilities. On the other hand, through industrial policies, fiscal policies, land policies, and copyright policies, large-scale equipment manufacturers are motivated to disseminate knowledge to other entities in the park.

Industrial systems have the structural characteristics of multidimensional heterogeneous nesting, and each scale contains the heterogeneity of elements and the heterogeneity of structure, environment, and system functions. This paper uses the framework of system theory to construct a variety of heterogeneity and study its mechanism in the evolution of industrial systems. The improvement of quality and efficiency is the ultimate goal of the development and upgrading of the equipment manufacturing industry. The quality of industrial inputs is critical to the improvement of industrial production efficiency. This paper uses industrial input quality indicators to study the impact of quality differences on the evolution of green total factor productivity in equipment manufacturing. Using quality indicators for input and output to achieve logical consistency can solve the relationship between quality and quality and make quality analysis more accurate.

\section{Data Availability}

No data were used to support this study.

\section{Conflicts of Interest}

The authors declare that they have no conflicts of interest.

\section{Acknowledgments}

This work was supported by the National Natural Science Foundation of China (71771083) and Research Projects of Hunan Provincial Department of Education of China (20A298).

\section{References}

[1] H.-L. Guan, "An evolution model for regional collaborative innovation under the perspective of complex network," Journal of Intelligent \& Fuzzy Systems, vol. 31, no. 3, pp. 1319-1328, 2016.

[2] F. Piccinno, R. Hischier, A. Saba, D. Mitrano, S. Seeger, and C. Som, "Multi-perspective application selection: a method to identify sustainable applications for new materials using the example of cellulose nanofiber reinforced composites," Journal of Cleaner Production, vol. 112, no. 1, pp. 1199-1210, 2016.

[3] X. Li, "The innovative management mechanism for the ecological environment of photovoltaic new energy industrial clusters," Light and Engineering, vol. 25, no. 3, pp. 37-43, 2017.

[4] D. V. Hertem, W. Leterme, G. Chaffey et al., "Substations for future HVdc grids: equipment and configurations for connection of HVdc network elements," IEEE Power and Energy Magazine, vol. 17, no. 4, pp. 56-66, 2019.

[5] A. E. Karlik and V. V. Platonov, "Cross-industry spatially localized innovation networks," Economy of Region, vol. 1, no. 4, pp. 1218-1232, 2016.

[6] Q. Zhong, "Evolution model and simulation of industrial cluster innovation network based on complex network," $\mathrm{C} e$ Ca, vol. 42, no. 2, pp. 740-745, 2017.

[7] J. Jin-Bo and P. Xu-Dong, "Leakage and stiffness characteristics of bionic cluster spiral groove dry gas seal," Chinese Journal of Mechanical Engineering, vol. 31, no. 2, pp. 148-158, 2018.

[8] F. Chang, G. Zhou, X. Xiao, C. Tian, and C. Zhang, "A function availability-based integrated product-service network model for high-end manufacturing equipment," Computers \& Industrial Engineering, vol. 126, pp. 302-316, 2018.

[9] R. C. Yam, "Does country-level R\&D efficiency benefit from the collaboration network structure?" Research Policy: A Journal Devoted to Research Policy, Research Management and Planning, vol. 45, no. 4, pp. 770-784, 2016.

[10] Y. Lu, G. Shailer, and M. Wilson, "Corporate political donations: influences from directors' networks," Journal of Business Ethics, vol. 135, no. 3, pp. 461-481, 2016.

[11] Z. Ke, "Research on hemingway inferiority complex from the perspective of Adler's compensation mechanism," Agro Food Industry Hi Tech, vol. 28, no. 1, pp. 764-767, 2017.

[12] H. E. Fan, J. M. Zhang, and T. H. Gao, "Evolution mechanism of the topological structure during solid-liquid phase transition of InGaAs crystal," Chinese Ence Bulletin, vol. 62, no. 7, pp. 693-699, 2017.

[13] J. L. Tangen and C. S. Cashwell, "Touchstones of connection: a concept mapping study of counselor factors that contribute to relational depth," The Journal of Humanistic Counseling, vol. 55, no. 1, pp. 20-36, 2016.

[14] S. V. Dronov and E. A. Evdokimov, "Post-hoc cluster analysis of connection between the forming characteristics," Model Assisted Statistics and Applications, vol. 13, no. 2, pp. 183-195, 2018.

[15] D. Dilawaer and Hiroki, "Simple algorithms for selecting an energy-efficient server in a cluster of servers," International Journal of Communication Networks and Distributed Systems: IJCNDS, vol. 21, no. 1, pp. 1-25, 2018.

[16] Y. Hong, P. Can, Y. Xiaona, and L. Ruixue, "Does change of industrial structure affect energy consumption structure: a study based on the perspective of energy grade calculation," Energy Exploration \& Exploitation, vol. 37, no. 1, pp. 579-592, 2019.

[17] S. Kergroach, "National innovation policies for technology upgrading through GVCs: a cross-country comparison," Technological Forecasting and Social Change, vol. 145, pp. 258-272, 2019.

[18] S. Fraser-Bell, R. Symes, and A. Vaze, "Hypertensive eye disease: a review," Clinical \& Experimental Ophthalmology, vol. 45, no. 1, pp. 45-53, 2017. 
[19] W. Gao and Z. Zhu, "The technological progress route alternative of carbon productivity promotion in China's industrial sector," Natural Hazards, vol. 82, no. 3, pp. 1803-1815, 2016.

[20] S. Tiley Jaimie, "A century of progress at the materials and manufacturing directorate throughout its 100-year history, the Materials and Manufacturing Directorate (ML) has conducted groundbreaking critical research programs responsible for a multitude of technological advances," $A d$ vanced Materials \& Processes, vol. 175, no. 2, pp. 24-27, 2017.

[21] A. K. Yetisen, A. F. Coskun, G. England et al., "Art on the nanoscale and beyond," Advanced Materials, vol. 28, no. 9, pp. 1724-1742, 2016.

[22] J. Li, K. F. See, and J. Chi, "Water resources and water pollution emissions in China's industrial sector: a green-biased technological progress analysis," Journal of Cleaner Production, vol. 229, pp. 1412-1426, 2019.

[23] T. Mertelmeier and H. M. Hofmann, "Consistent cluster model description of the electromagnetic properties of lithium and beryllium nuclei," Nuclear Physics, vol. 459, no. 2, pp. 387-416, 2016.

[24] H. Chen, P. He, C. X. Zhang et al., "Efficiency of technological innovation in China's high tech industry based on DEA method," Journal of Interdisciplinary Mathematics, vol. 20, no. 6-7, pp. 1493-1496, 2017. 